\title{
List of reviewers
}

The Editors would like to thank the following individuals who kindly agreed to serve as referees for the Journal.

Balestrino, Alessandro

Belleflamme, Paul

Bishop, Paul

Canals-Cerda, Jose

Chen, Chien-Ping

Chi, Christina Geng-Qing

Collins, Alan

Cox, Joe

Dass, Mayukh

Davis, Charlene

del Bosque, Ignacio Rodriguez

Delaney, Liam

Earl, Peter

Einav, Liran

Elberse, Anita

Fernandez-Blanco, Victor

Halicioglu, Ferda

Hand, Chris

Houser, Daniel

$\mathrm{Hu}$, Weimin

Ioannou, Ioannis

Jackson, Karen

Livingston, Jeffrey

Luksetich, William

Maddison, David
Marco-Serrano, Francisco

McKenzie, Jordi

Montmarquette, Claude

Nicolau, Juan

Ohashi, Hiroshi

Pesando, James

Phillips, Ronnie J.

Ravid, S. Abraham

Regner, Tobias

Sargeant, Adrian

Sedgwick, John

Snowball, Jeanette

Spaenjers, Christophe

Sproule, Robert

Stamm, Brad

Tohmo, Timo

Urrutiaguer, Daniel

Walls, W. David

Wildman, Steven

Willis, Kenneth

Wolff, Francois-Charles

Worthington, Andrew

Zanola, Roberto

Zieba, Marta 